This is an Accepted Manuscript of an article published by Taylor \& Francis in Pharmaceutical Development and Technology on 24/08/17, available online:

http://www.tandfonline.com/doi/full/10.1080/10837450.2017.1364267?needAccess=true 


\section{The Use of Albumin Solid Dispersion to Enhance the Solubility of Unionisable Drugs}

Mouhamad Khoder ${ }^{\mathrm{a} *}$, Hamdy Abdelkader ${ }^{\mathrm{b}}$, Amr ElShaer ${ }^{\mathrm{a}}$, Ayman Karam ${ }^{\mathrm{c}}$, Mohammad Najlah $^{\mathrm{d}}$, Raid G. Alany ${ }^{\mathrm{a}, \mathrm{e}}$

a Drug Discovery, Delivery and Patient Care (DDDPC) Theme, School of Life Sciences, Pharmacy and Chemistry, Kingston University London, Kingston Upon Thames, United Kingdom.

${ }^{\mathrm{b}}$ Department of Pharmaceutics, Faculty of Pharmacy, Minia University, Minia, Egypt.

'Institute of Chemistry of Poitiers : Materials and Natural Resources, Catalysis and unconventional media's Team University of Poitiers, France

${ }^{d}$ Faculty of Medical Science, Anglia Ruskin University, Bishops Hall Lane, Chelmsford CM1 1SQ, United Kingdom

'School of Pharmacy, The University of Auckland, Auckland, New Zealand

*Corresponding author

Mouhamad Khoder, Ph.D.

Drug Discovery, Delivery and Patient Care (DDDPC) Theme,

School of Life Sciences, Pharmacy and Chemistry,

Kingston University London, Penrhyn Road,

Kingston Upon Thames, Surrey KT1 2EE, UK,

Tel +442084179000

e-mail: $\underline{\text { m.khoder@ kingston.ac.uk, }}$

mouhamad.khoder@gmail.com 


\section{The use of albumin solid dispersion for enhanced un-ionisable drugs solubility}

In this study, solid dispersions of prednisolone (PRD) and bovine serum albumin (BSA) were prepared by spray drying and freeze drying methods using a PRD:BSA solution (20:1 Molar Ratio (MR)). PRD-BSA dispersed mixtures were characterised by scanning electron microscopy (SEM), and powder X-ray diffraction (XRD), and differential scanning calorimetry (DSC). PRD-BSA physical and dispersed mixtures showed significantly higher solubility in water than that of unprocessed drug. Enhancement factor of six was obtained in both physical mixture and solid dispersion solubility studies. In-vitro dissolution and release studies under physiological conditions showed an immediate release of PRD from the solid dispersions, with almost $90 \%$ of the drug dissolved in the first $10 \mathrm{~min}$. PRD was immediately released from BSA binding complex. This study demonstrates the potential for the use of BSA to enhance the solubility and dissolution rate, hence bioavailability, of the unionisable drugs.

Keywords: bovine serum albumin, prednisolone, solid dispersion, solubility enhancer, dissolution rate.

\section{Introduction}

The recent drug discovery techniques have led to an increasing number of drugs that are poorly soluble in water. It is estimated that $40 \%$ of the approved drugs and almost $90 \%$ of drugs candidates are poorly soluble (Kalepu and Nekkanti 2015). For this reason, the development of new strategies to enhance drug solubility remains one of the major research areas in drug discovery and development process (Bellantone 2014, Bosselmann and Williams 2012). An approach to enhance the solubility of ionisable drugs is by adjusting the $\mathrm{pH}$ of the solvent or by forming soluble salts (Kalepu and Nekkanti 2015, Owen 2013, Serajuddin 2007). Howover, the $\mathrm{pH}$ has less pronounced impact on the solubility of the unionisable drugs; consequently, they are insoluble in various parts of GIT. Therefore, enhancing the solubility of this drug category is considered more challenging (Bellantone 
2014, Bosselmann and Williams 2012). Several approaches have been developed to enhance unionisable drugs solubility including, but not limited to, size reducing approach, particle engineering, micellization, and cyclodextrins and polymers complexation (Cavallari et al. 2005, Chen et al. 2011, Del Valle 2004, Junghanns and Müller 2008, Strickley 2004).

In a recently published work, we have described the use of albumin as solubility enhancer of ionisable drugs using indomethacin as drug model. An enhancement factor of 100,000 was obtained after formulating indomethacin in BSA solid dispersions. The solubility enactment of indomethacin in presence of BSA is thought to be mainly due to the formation of salt bridges between the drug and the albumin molecules (Khoder et al. 2016). However, the solubilizing properties of albumin are not limited to its ability to form salts with ionisable molecules but also to its remarkable ability to form reversible binding complexes with hydrophobic drugs (Ghuman et al. 2005, Urien et al. 2007). Indeed, albumin displays two main sites that bind the ligands mainly by hydrophobic and electrostatic interactions. Several secondary binding sites are also available that increase the number of bound molecules (Evans 2002). The hydrophobic molecules of medium size (100-600 Da) are amongst the substances that showed the highest affinity to albumin. The interaction forces involved in the binding process are mainly hydrophobic, Van der Waals force and hydrogen bonding interactions (Peters Jr 1995, Shi et al. 2013b, Urien, Tillement and Barré 2007).

Bovine serum albuin (BSA) has been widely used in numerous pharmaceutical and medical applications thanks to its stability, biocompatibility and low immunogenicity (Elzoghby et al. 2012, Khoder et al, 2016). The aim of this study is to investigate the potential use of the BSA to enhance the solubility of the unionisable drugs using PRD as a model drug. Prepared by freeze drying and spray drying techniques, PRD-BSA solid dispersions are characterised by SEM, XRD, and DSC. PRD solubility is evaluated in water in the presence of increased 
amounts of BSA. In addition, the dissolution rates and release profiles are established under physiological conditions.

\section{Materials and Methods}

\section{Materials}

PRD was provided by Acros organics (USA). BSA lyophilised powder was purchased from sigma-Aldrich (UK). Methanol, ethanol, trichloroacetic acid (TCAA), and all the other materials and reagents used in protein precipitation, analytical methods, and medium preparation were of analytical grade of purity and were all purchased from sigma-Aldrich (UK).

\section{Methods}

\section{Solid dispersions preparation}

A specified amount $(0.12 \mathrm{~g})$ of PRD was dissolved in $5 \mathrm{ml}$ ethanol and gradually added to $100 \mathrm{ml} \mathrm{BSA}$ solution $(1 \% \mathrm{w} / \mathrm{v})$. The resulting PRD-BSA hydro-alcoholic solution (20:1 PRD:BSA MR) was frozen in liquid nitrogen and freeze-dried for $72 \mathrm{~h}$ using (VirTis BenchTop Pro, UK). Alternatively, for the preparation of spray-dried formulation, the resulting PRD-IND solution was spray-dried using Buchi spray dryer (Buchi, Switzerland) under the following conditions: feeding rate of $1 \mathrm{~mL} / \mathrm{min}$, inlet temperature of $120{ }^{\circ} \mathrm{C}$, and outlet temperatures of $50-60{ }^{\circ} \mathrm{C}$.

The resulting PRD-BSA solid dispersions were named as showed in the table 1:

[Table 1 near here] 


\section{Differential scanning calorimetry (DSC)}

DSC thermograms of PRD, BSA, PRD:BSA physical mixture, SD-sd and FD-sd were obtained using differential scanning calorimeter (Mettler Toledo, DSC822e, UK). Samples (2-5mg) were weighed and crimp-sealed in aluminium pans with the led pierced. The samples were heated under nitrogen gas flow $(20 \mathrm{~mL} / \mathrm{min})$ over a temperature range of $25{ }^{\circ} \mathrm{C}$ to 300 ${ }^{\circ} \mathrm{C}$ and at a heating rate of $10{ }^{\circ} \mathrm{C} / \mathrm{min}$. The obtained thermograms were analysed using STAReSW 10.00 software.

\section{$X$-ray Diffractometry}

Diffraction patterns of the samples outlined above (under DSC section) were obtained using Bruker diffractometer (Bruker AXS D8 Advance; Bruker Corporation, Billerica, MA, USA). The diffractometer operated at room temperature and at $40 \mathrm{kV}$. Samples were scanned from from $2^{\circ}$ to $45^{\circ} 2 \theta$ at a step size of $0.1^{\circ}$ and collected data were analysed using DIFFRAC plus XRD commander software (Bruker Corporation).

\section{Scanning electron microscopy}

The morphology of the PRD, BSA, FD-sd and SD-sd samples were examined using Zeiss Evo50 electron microscope (Oxford instrument, UK). Prior to imaging and in order to enable electricity conduction, samples were coated with a fine layer of gold using a sputter coater (Polaron SC500, Polaron Equipment, Watford, UK) at $20 \mathrm{~mA}$ for $3 \mathrm{~min}$ at low vacuum and in the presence of argon gas (Polaron Equipment, Watford, UK). The microscope was operated at an accelerating voltage of $30 \mathrm{kV}$ under low-vacuum mode.

\section{Equilibrium solubility studies}

The equilibrium solubility of PRD was determined using saturation shake-flask method by adding an excess amount of drug $(50 \mathrm{mg})$ to $1 \mathrm{~mL}$ distilled water containing increased 
amounts of BSA. The final concentrations of BSA were $(0,0.3,0.6,0.9,1.8$ and $3.6 \mathrm{mM})$. To assess the solubility of PRD formulated in solid dispersions, increased amount of FD-sd and SD-sd were added to $1 \mathrm{~mL}$ distilled water to produce final concentrations of BSA that were equal to those mentioned above. The samples were then shaken at $25{ }^{\circ} \mathrm{C}$ for $24 \mathrm{~h}$ to reach equilibrium, centrifuged for $3 \mathrm{~min}$ at 10,000 rpm (RCF: $\left.12.27 \times 10^{4}\right)$ (Thermo Scientific, Germany), and the supernatants were collected and filtered through $0.45 \mu \mathrm{m}$ Milipore filters). The drug concentrations in the obtained filtrates were determined using the HPLC analysis method as detailed below.

\section{Analytical method}

The chromatographic analysis was performed using PerkinElmer HPLC system (USA) which consisted of a degasser, a pump, an auto-sampler, and a UV detector set at $254 \mathrm{~nm}$. The separation was carried out using a HyperClone ${ }^{\mathrm{TM}}$ analytical column $(4.6 \times 150 \mathrm{~mm}, \mathrm{C} 18,5 \mu \mathrm{m}$, $130 \AA)$ and an isocratic mobile phase consisted of methanol (70\% v/v) and water $(30 \% \mathrm{v} / \mathrm{v})$. The flow rate was $1.0 \mathrm{~mL} / \mathrm{min}$ and the simple injection volume was $10 \mu 1$.

BSA-free samples were adequately diluted with mobile phase and the drug concentration was determined using a calibration standard obtained in mobile phase. The HPLC method was linear in the mbile phase over a range of $7.5 \mu \mathrm{g} / \mathrm{mL}$ to $0.5 \mathrm{mg} / \mathrm{mL}$ with a correlation coefficient (r2) of 0.998. For BSA-containing samples, samples were adequately diluted with water to reach BSA concentration in the calibration curve $(0.03 \mathrm{mM})$ and the concentration of drug was determined using a calibration standard prepared in BSA solution $(0.03 \mathrm{mM})$. In BSA containing samples, the HPLC method was linear over a range of $25 \mu \mathrm{g} / \mathrm{mL}$ to 0.5 $\mathrm{mg} / \mathrm{mL}$ with a correlation coefficient (r2) of 0.996. Prior injection in HPLC system, PRD extraction from all BSA-containing samples was realised. Briefly, $0.2 \mathrm{~mL}$ sample was placed in $1.5 \mathrm{~mL}$ Eppendorf tube and $0.8 \mathrm{~mL}$ TCAA solution $(10 \% \mathrm{w} / \mathrm{v})$ was added and vortex- 
mixed for $2 \mathrm{~min}$. Samples were left for $30 \mathrm{~min}$ and centrifuged for $3 \mathrm{~min}$ at 10,000 rpm $\left(\mathrm{RCF}: 12.27 \times 10^{4}\right)($ Thermo Scientific, Germany). The supernatants were filtrated using 0.45 $\mu \mathrm{m}$ Milipore filters and diluted 5 times with the mobile phase before being injected into the HPLC system. The extraction recovery (\%) of PRD from BSA solution was $(92.16 \pm 11)$.

\section{Dissolution studies and release studies}

In-vitro dissolution profiles of pure PRD, SD-sd, and FD-sd were established using USP dissolution apparatus 2-Paddle (CALEVA, UK). The dissolution study was conducted in 900 $\mathrm{ml}$ of phosphate buffer $\mathrm{pH} 7$, at $37 \pm 0.5^{\circ} \mathrm{C}$ using a stirring rate of $100 \mathrm{rpm}$. The powders (equivalent to $40 \mathrm{mg}$ PRD) were dispersed in the dissolution medium and aliquots of $2 \mathrm{ml}$ were withdrawn at $0,5,10,15,20,30,45,60 \mathrm{~min}$. Aliquots were filtered through $0.45 \mu \mathrm{m}$ Milipore filters before being analysed using UV spectrometer (JENWAY, UK) at $254 \mathrm{~nm}$.

In order to evaluate the drug release from albumin complex, in-vitro drug release was performed using Franz diffusion cells (LOGAN Instruments Corp, USA). Cellulosic synthetic membranes (Cut-off $12 \mathrm{KD}$, Medicell Membrane Ltd, UK) were soaked in distilled water for $24 \mathrm{~h}$ before being mounted between the two compartments of Franz cells. The cut-off of used membrane allows the diffusion of the free drug only. Receptor's compartment was filled with $12 \mathrm{~mL}$ PBS (pH 7) and was continuously homogenized using stirring magnetic bars and kept at $37{ }^{\circ} \mathrm{C}$. The donner compartments were filled with $2 \mathrm{~mL}$ of PRD, SD-sd, and FD-sd aqueous solutions $(0.05 \% \mathrm{w} / \mathrm{v})$ and covered with Parafilm to avoid evaporation. The air bubbles formed underneath the membrane were removed via the side arm by carefully tilting the Franz cell. Serial sampling of $1 \mathrm{~mL}$ was performed after $0.0 .5,1,2,3,4,5$, and $6 \mathrm{~h}$ and fresh receptor liquid was added to receptor compartment to replace the buffer. The amount of diffused drugs was determined spectrophotometrically. As BSA molecules could not penetrate through the membrane pore, no extraction step was needed. The UV method was 
validate against the HPLC reported in the paper.

\section{Statistical analysis}

Statistical significance was determined using the one-way analysis of variance (ANOVA) and Student's t-tests as appropriate. All experiments were performed in triplicate and values were expressed as the mean \pm standard deviation. Values of $P<0.05$ were considered statistically significant.

\section{Results}

\section{Solid dispersions characterisation}

The surface morphology of PRD, BSA, SD-sd and FD-sd powders was examined using scanning electron microscopy (Fig. 1). While PRD SEM image revealed crystals of different shapes and size (Fig. 1a), those of BSA showed big plate-like chunks (Fig. 1b). On the other hand, FD-sol powder showed ribbon-like shape without any evidence of crystallinity (Fig. 1d). SD-sol displayed rounded microparticles with an average size ranging between 0.2 to 2 $\mu \mathrm{m}$ and a smooth holed surface (Fig. 3c).

[Figure 1 near here]

Fig. 2 shows the DSC thermograms of PRD, BSA, PRD-BSA:PM, SD-sd and FD-sd. PRD displayed a sharp endothermic peak at $245{ }^{\circ} \mathrm{C}$ which assigned to its melting point (Couto et al. 2014, Huanbutta et al. 2016, Veiga et al. 1985). The same peak was also observed in the PRD-BSA physical mixture thermogram indicating the crystalline state of the drug. However, this endothermic peak disappeared completely from the thermograms of SD-sd and Fd-sd (Fig. 2) suggesting the dispersion of PRD in its amorphous form within the BSA matrix. Interestingly, a small exothermic peak was observed on the solid dispersions thermograms at 
145-150 ${ }^{\circ} \mathrm{C}$ that could be attributed to a partial recrystallization (polymorphic phase transition) of PRD under the DSC analysis conditions; the fact that might confirm the amorphous state of the drug (Veiga, Cadorniga and Lozana 1985).

[Figure 2 near here]

The solid state of PRD in the BSA matrix was also observed by XRD technique. The XRD pattern of raw and processed PRD and BSA are shown in Fig. 3.

[Figure 3 near here]

While the diffraction spectrum of BSA showed a typical pattern of amorphous structure, the PRD diffractogram revealed several sharp high-intensity peaks indicating its crystalline state (Huanbutta, Sangnim, Limmatvapirat, Nunthanid and Sriamornsak 2016, Li et al. 2007). Similar characteristic peaks were observed in the XRD pattern of PRD:BSA-PM (Fig. 3). However, those diffraction peaks disappeared completely from the XRD patterns of both spray-dried and freeze-dried solid dispersions (Fig. 3). These results are in a good agreement with those obtained with DSC.

\section{Solubility studies}

The drug solubility was firstly assessed in water in presence of increased amount of BSA. Fig. 4a shows the concentrations of dissolved PRD $(\mathrm{mg} / \mathrm{L})$ as a function of BSA concentrations $(\mathrm{mM})$. The addition of increased amounts of BSA resulted in significant and continuous increases in PRD solubility $(\mathrm{P}<0.05)$. The initial solubility of $\mathrm{PRD}$, in pure water, 
was around $200 \mathrm{mg} / \mathrm{L}$, which is in correspondence with the literature (Solubility Data 2003). Interestingly, PRD solubility increased significantly to the range of $1000 \mathrm{mg} / \mathrm{L}$; i.e. enhancement factor of about 5.34, when the BSA was added at a concentration of $3.6 \mathrm{mM}$ (24\%w/w) (Fig. 3a). The phase solubility diagram, obtained by plotting the PRD molar concentration against BSA molar concentration, revealed a linear relationship that is classified as "A $\mathrm{L}_{\mathrm{L}}$-type" (Fig. $4 \mathrm{~b}$ ). Based on the curve trend-line slope values $(<1)$, it is assumed that a 1:1 stoichiometry complex was formed (Higuchi 1965, Shi, Zhu, Wang, Chen and Shen 2013b). The binding constant $\left(\mathrm{K}_{1: 1}\right)$ has been determined from the solubility diagram as following:

$\mathrm{K} 1: 1=$ slope $/\left[\mathrm{S}_{0}(1-\right.$ slope $\left.)\right]$

Where $\left(\mathrm{S}_{0}\right)$ is drug initial solubility of the drug (Loftsson et al. 2005).

The estimated association constant $\left(\mathrm{K}_{1: 1}\right)$ was in the order of $2 * 10^{3} \mathrm{~mole}^{-1}$, which is in accordance with the results previously reported in the literature (Frey and Frey 1984, Legler and Benet 1986, Rocci et al. 1982). However, in a recent study, higher association constant value has been reported; $\sim 10^{6}$ mole $^{-1}$, indicating a stronger binding interaction between PRD and BSA (Shi, Zhu, Wang, Chen and Shen 2013b). However, PRD:BSA binding was carried out in TRIS buffer; this could interfere favourably in the formation of hydrogen bonds and yield a higher complexation extent.

[Figure 4 near here]

PRD solubility was then assessed after the preparation of the PRD:BSA solid dispersions. The solid dispersions were prepared by both freeze-draying and spray-drying techniques using a solution of (20:1) PRD:BSA molar ratio; 20 folds higher than the binding complex 
stoichiometry (1:1). Fig. 5 shows the dissolved amounts of PRD (mg/L) as a function of BSA concentrations (mM) for PRD:BSA PM, SD-sd and FD-sd. The initial solubility of freezedried and spray-dried PRD, with no BSA added, was similar to that of unprocessed PRD. Similarly, formulating PRD in BSA solid dispersions did not lead to a significant enhancement in PRD solubility compared to that obtained by PRD:BSA physical mixture $(P>0.05)($ Fig 5).

[Figure 5 near here]

\section{Dissolution and release studies}

Fig. 6a shows the dissolution profiles of PRD, SD-sd and FD-sd in phosphate buffer $(\mathrm{pH} 7)$ at $37{ }^{\circ} \mathrm{C}$. The dissolution rate of unprocessed PRD was relatively low with almost $15 \%$ and $60 \%$ dissolved after $10 \mathrm{~min}$ and $1 \mathrm{~h}$, respectively. On the other hand, formulating PRD in BSA solid dispersions allowed an instant dissolution with almost $90 \%$ dissolved within the first 10 minutes.

Franz diffusion cell was used to evaluate the drug release rate from BSA complex after the dissolution step using phosphate buffer $(\mathrm{pH} 7)$ as a release medium and a semi-permeable membrane that allows the diffusion of the free PRD molecules only. The released amount is the amount of drug diffused through the cellulosic membrane to the receptor compartment. Fig. $6 \mathrm{~b}$ shows that the PRD release rates from BSA complex were comparable to those obtained from PRD aqueous solution $(P>0.05)$ with approximately 50\% PRD diffused to the receptor compartment after $6 \mathrm{~h}$.

[Figure 6 near here] 


\section{Discussion}

In a recent study, we demonstrated the possible use of BSA as solubility enhancer for indomethacin as ionisable drugs model, where the solubility was improved up to $10^{5}$ times after formulating it in BSA solid dispersion (Khoder et al, 2016). This spectacular solubility enhancement was mainly attributed to the formation of salts bridges between indomethacin carboxylate groups and BSA amino groups. In this study, we investigated the potential use of BSA solid dispersion approach to enhance the solubility of unionisable drugs using PRD as drug model. Unionisable drugs are not expected to form salts with BSA molecules, yet they might bind to albumin molecules and form a reversible drug-albumin binding complex of higher solubility (Ghuman, Zunszain, Petitpas, Bhattacharya, Otagiri and Curry 2005, Urien, Tillement and Barré 2007). Additionally, converting the drug into a molecular and/or amorphous state that is homogeneously dispersed within the hydrophilic matrix provided by BSA might actively contribute in the enhancement of drug solubility and dissolution rate (Graeser et al. 2010, Hancock and Zografi 1997, Huang and Dai 2014, Khoder et al, 2016). PRD:BSA solid dispersions were obtained using two different particle engineering technologies; spray-drying and freeze-drying. SEM, DSC, and XRD results (Fig. 1-3) revealed the amorphous or/and molecular state of PRD within the BSA matrix. The surface morphology of spray dried powder displayed rounded and smooth microparticles with a size ranging between 0.3 and $3 \mu \mathrm{m}$ and a single hole at the surface (Fig.1c). The formation of this single indentation on particles surface could be explained by the outbreak of the skin-like outer shell formed on the droplet surface due to high pressure generated during the heating process (Khoder et al, 2016, Walzel and Furuta 2011). Unprocessed PRD displayed a poor solubility in water that could be attributed to its crystalline state (Fig. 1-3) and lipophilic properties. However, PRD aqueous solubility has been significantly and steadily enhanced by 
increasing amounts of BSA (Fig 4). PRD solubility enhancement might be explained by the spontaneous formation of a soluble 1:1 binding complex between PRD and BSA molecules, where each PRD molecule was accommodated into the hydrophobic cavity of the subdomain IIIA (site II) of BSA (Shi, Zhu, Wang, Chen and Shen 2013b). The interaction forces involved in the binding process are mainly hydrophobic, Van der Waals force and hydrogen bonding interactions (Shi, Zhu, Wang, Chen and Shen 2013b). The extent of PRD solubility enhancement directly relates to its binding affinity to BSA and the solubility of the resulting complex. The PRD-BSA solid dispersions were prepared by either spray-drying or freezedrying of a (20:1 MR) PRD:BSA solution. The aim of increasing the PRD:BSA molar ratio from 1:1 (the binding ratio obtained in the physical mixture) to 20:1 was to allow more molecular interactions between PRD and BSA to take place during the drying process and, thus, improve further the solubility enhancement. Formulating PRD:BSA solution into solid dispersions by both spray-drying and freeze-drying techniques did not result in any significant additional solubility enhancement compared to that obtained by the physical mixture study (Fig. 5). This is mainly because the complexation capacity of BSA molecule is limited to one PRD molecule only. This result highlights the fact that the formation of binding complex is the main mechanism behind the solubility enhancement of PRD. Furthermore, the two drying techniques used to form the solid dispersions had no impact on BSA stability, i.e., BSA molecules seemed to refold successfully in water to form the PRD:BSA complex. Compared to previous reports, the solubility enhancement achieved by BSA solid dispersion of PRD has found to be significantly higher than that of PVP spry-dried solid dispersion, similar to that of the $\gamma$-cyclodextrin solid dispersion, and lower than that obtained by $\beta$-cyclodexrin solid dispersion (Couto, Vieira, Florindo, Videira and CabralMarques 2014, Fukuda et al. 1986, Ghuzlaan et al. 2009). However, due to its high molecular weight, BSA concentrations used for solubility enhancement in this study were lower than 
those of cyclodexrins. The amorphous and consistent dispersion of PRD within the hydrophilic matrix of BSA led to a significant enhancement in the drug dissolution (Fig 6. a) (Graeser, Patterson, Zeitler and Rades 2010). Besides, the formation of the molecular binding complex between PRD and BSA molecules might also help protect the molecular state of PRD in BSA matrix. Therefore, the step of drug dissolution might be bypassed, and an immediate solution might be obtained. Franz diffusion cell was used in this study to evaluate the drug release rate from BSA complex after the dissolution step. Franz diffusion cell was used in in this study to evaluate the drug release rate from BSA complex after the dissolution step. For this reason, the membrane pore size was selected in such a way that it allows the diffusion of the free drug only. The experiment results demonstrated that albumin molecule released immediately the bond molecules (Fig. 6b). Indeed, Van der Waals and hydrogen bonding interaction are thought to be the main intramolecular forces binding PRD to BSA molecules (Shi et al. 2013a). The weak nature of these intramolecular forces was reflected on the relatively low association constant reported in this study. The concentration gradient and the osmotic pressure on the both sides of the diffusion membrane are thought to be high enough to disassociate PRD-BSA complex and immediately release PRD molecules through the cellulosic membrane. This immediate release is a key factor to achieve rapid in-vivo absorption, hence improved bioavailability.

\section{Conclusion}

In this study, we demonstrated the possible use of BSA particle engineering technique to enhance the solubility of unionisable drugs using PRD as drug model. In physical mixture, a significant enhancement in PRD solubility dependent on BSA concentrations was observed. PRD:BSA solid dispersions prepared by both freeze-drying and spray-drying methods produced an enhancement in drug solubility comparable to that of physical mixture. PRD was 
found to transform into the amorphous/molecular state within the BSA hydrophilic matrix.

This produced a significant increase in the drug dissolution rate compared to that of the free drug. Additionally, an immediate release of PRD from BSA binding complex was also reported.

\section{Disclosure of interest}

The authors report no conflicts of interest

\section{References}

Bellantone AR. 2014. Fundamentals of Amorphous Systems: Thermodynamic Aspects. In: Amorphous Solid Dispersions: Theory and Practice. New York, NY: Springer New York. p. 3-34. Bosselmann S, Williams RO. 2012. Route-Specific Challenges in the Delivery of Poorly Water-Soluble Drugs. In: Formulating Poorly Water Soluble Drugs. New York, NY: Springer New York. p. 1-26. Cavallari C, Albertini B, Rodriguez L, Rabasco AM, Fini A. 2005. Release of indomethacin from ultrasound dry granules containing lactose-based excipients. Journal of Controlled Release. 1/20/;102:39-47.

Chen H, Khemtong C, Yang X, Chang X, Gao J. 2011. Nanonization strategies for poorly water-soluble drugs. Drug Discovery Today. 4//;16:354-360.

Couto AS, Vieira J, Florindo HF, Videira MA, Cabral-Marques HM. 2014. Characterisation of DM- $\beta$ cyclodextrin:prednisolone complexes and their formulation as eye drops. Journal of Inclusion Phenomena and Macrocyclic Chemistry. 2014//;80:155-164.

Del Valle EMM. 2004. Cyclodextrins and their uses: a review. Process Biochemistry. 5/31/;39:10331046.

Elzoghby AO, Samy WM, Elgindy NA. 2012. Albumin-based nanoparticles as potential controlled release drug delivery systems. Journal of Controlled Release. 1/30/;157:168-182.

Evans TW. 2002. Review article: albumin as a drug-biological effects of albumin unrelated to oncotic pressure. Alimentary Pharmacology \& Therapeutics.16:6-11.

Frey FJ, Frey BM. 1984. Altered Plasma Protein-Binding of Prednisolone in Patients With the Nephrotic Syndrome. American Journal of Kidney Diseases. 1984/03/01;3:339-348.

Fukuda N, Higuchi N, Ohno M, Kenmochi H, Sekikawa H, Takada M. 1986. Dissolution Behavior of Prednisolone from Solid Dispersion Systems with Cyclodextrins and Polyvinylpyrrolidone. CHEMICAL \& PHARMACEUTICAL BULLETIN.34:1366-1369.

Ghuman J, Zunszain PA, Petitpas I, Bhattacharya AA, Otagiri M, Curry S. 2005. Structural Basis of the Drug-binding Specificity of Human Serum Albumin. Journal of Molecular Biology. 10/14/;353:38-52. Ghuzlaan A, Al Omari MM, Al-Sou'od KA. 2009. Prednisone/Cyclodextrin Inclusion Complexation: Phase Solubility, Thermodynamic, Physicochemical and Computational Analysis. Journal of Solution Chemistry.38:83-94.

Graeser KA, Patterson JE, Zeitler JA, Rades T. 2010. The Role of Configurational Entropy in Amorphous Systems. Pharmaceutics.

Hancock BC, Zografi G. 1997. Characteristics and Significance of the Amorphous State in Pharmaceutical Systems. Journal of Pharmaceutical Sciences. 1997/01/01;86:1-12. 
Higuchi T. 1965. Advances in Analytical Chemistry and Instrumentation, Chapter 4. Phase Solubility Studies.117-212

Huanbutta K, Sangnim T, Limmatvapirat S, Nunthanid J, Sriamornsak P. 2016. Design and characterization of prednisolone-loaded nanoparticles fabricated by electrohydrodynamic atomization technique. Chemical Engineering Research and Design. 5//;109:816-823.

Huang Y, Dai W-G. 2014. Fundamental aspects of solid dispersion technology for poorly soluble drugs. Acta Pharmaceutica Sinica B. 2//;4:18-25.

Junghanns J-UAH, Müller RH. 2008. Nanocrystal technology, drug delivery and clinical applications. International Journal of Nanomedicine.3:295-310.

Kalepu S, Nekkanti V. 2015. Insoluble drug delivery strategies: review of recent advances and business prospects. Acta Pharmaceutica Sinica B. 9//;5:442-453.

Khoder M, Abdelkader H, ElShaer A, Karam A, Najlah M, Alany RG. 2016. Efficient approach to enhance drug solubility by particle engineering of bovine serum albumin. International Journal of Pharmaceutics. 12/30/;515:740-748.

Legler UF, Benet LZ. 1986. The effect of prednisone and hydrocortisone on the plasma protein binding of prednisolone in man. European Journal of Clinical Pharmacology. 1986;30:51-55.

Li X-S, Wang J-X, Shen Z-G, Zhang P-Y, Chen J-F, Yun J. 2007. Preparation of uniform prednisolone microcrystals by a controlled microprecipitation method. International Journal of Pharmaceutics. 9/5/;342:26-32.

Loftsson T, Jarho P, Masson M, Jarvinen T. 2005. Cyclodextrins in drug delivery. Expert Opin Drug Deliv. Mar;2:335-351.

Owen IC. 2013. Salt Forms: Pharmaceutical Aspects. In: Encyclopedia of Pharmaceutical Technology, Third Edition. Taylor \& Francis. p. 3177-3187.

Peters Jr T. 1995. 3 - Ligand Binding by Albumin. In: All About Albumin. San Diego: Academic Press. p. 76-132.

Rocci ML, D'Ambrosio R, Johnson NF, JusKo WJ. 1982. Prednisolone binding to albumin and transcortin in the presence of cortisol. Biochemical Pharmacology. 1982/02/01;31:289-292.

Serajuddin ATM. 2007. Salt formation to improve drug solubility. Advanced Drug Delivery Reviews. 7/30/;59:603-616.

Shi J-h, Zhu Y-Y, Wang J, Chen J, Shen Y-J. 2013a. Intermolecular interaction of prednisolone with bovine serum albumin: Spectroscopic and molecular docking methods. Spectrochimica Acta Part A: Molecular and Biomolecular Spectroscopy. 2/15/;103:287-294.

Shi JH, Zhu YY, Wang J, Chen J, Shen YJ. 2013b. Intermolecular interaction of prednisolone with bovine serum albumin: spectroscopic and molecular docking methods. Spectrochim Acta A Mol Biomol Spectrosc. Feb 15;103:287-294.

Solubility Data. 2003. In: Handbook of Aqueous Solubility Data. CRC Press.

Strickley RG. 2004. Solubilizing Excipients in Oral and Injectable Formulations. Pharmaceutical Research.21:201-230.

Urien S, Tillement J-P, Barré J. 2007. The Significance of Plasma-Protein Binding in Drug Research. In: Pharmacokinetic Optimization in Drug Research. Verlag Helvetica Chimica Acta. p. 189-197.

Veiga MD, Cadorniga R, Lozana R. 1985. Thermal study of prednisolone polymorphs. Thermochimica Acta. 1985/11/20;96:111-115.

Walzel P, Furuta T. 2011. Morphology and Properties of Spray-Dried Particles. In: Modern Drying Technology. Wiley-VCH Verlag GmbH \& Co. KGaA. p. 231-294. 